\title{
Three ways to compute multiport inertance
}

\author{
$\begin{array}{lll}\text { G. D. McBain } & \text { S. G. Mallinson } & \\ & \text { B. R. Brown }\end{array}$ \\ Tom Gustafsson ${ }^{4}$
}

(Received 26 February 2019; revised 8 July 2019)

\begin{abstract}
The immediate impulse-response of a confined incompressible fluid is characterized by inertance. For a vessel with one inlet and outlet, this is a single quantity; for multiple ports the generalization is a singular reciprocal inertance matrix which acts on the port-impulses to give the corresponding inflows. The reciprocal inertance coefficients are defined by the boundary fluxes of potential flows. Green's identity converts these boundary fluxes to domain integrals of kinetic energy. For a system discretized with finite elements, a third method is proposed for computing reciprocal inertance coefficients which requires only the stiffness matrix and the solution vectors and no numerical differentiation.
\end{abstract}

DoI:10.21914/anziamj.v60i0.14058 gives this article, (c) Austral. Mathematical Soc. 2019. Published August 26, 2019, as part of the Proceedings of the 18th Biennial Computational Techniques and Applications Conference. ISSN 1445-8810. (Print two pages per sheet of paper.) Copies of this article must not be made otherwise available on the internet; instead link directly to the DOI for this article. 


\section{Contents}

1 Introduction

C141

2 Theory of hydraulic inertance

2.1 Short-time impulse-response of incompressible fluid . . . . C143

2.2 The multiport boundary value problem . . . . . . . . . C144

2.3 Reciprocal inertance matrix . . . . . . . . . . . C C144

2.4 Two ways to calculate reciprocal inertance . . . . . . . . C145

2.5 Properties of the reciprocal inertance matrix . . . . . . . C146

3 Discretization

3.1 A third way to compute reciprocal inertance . . . . . . C147

4 Implementation

C148

4.1 Pseudocode ..................... C148

4.2 Example: Asai's prototype bubble jet . . . . . . . . . . . C149

4.3 Convergence . . . . . . . . . . . . . . . . . . . C149

5 Conclusion

C151

\section{Introduction}

Microhydraulic devices often need analyses other than those traditional in industrial fluid mechanics. Many have a complicated topology; for example, the single-pass ink-jet printing chips discussed by Mallinson et al. [15] each have 640 nozzles per colour, and eleven chips are plumbed together across a page. Another characteristic of a microhydraulic device is small size; this keeps the Reynolds number low and the flow laminar or even creeping. The combination of hydraulic linearity and topological complexity makes circuit theory appealing [17]. The use of circuit theory is widespread, building on earlier work in fluidics [7], acoustics [18], and general systems theories [19]. 
Going back further, this approach can be seen as the reverse of the old 'waterpipe' analogy in which the 'flow' of electrons was likened to 'current' [11, e.g.].

The basic idea of microhydraulic circuit theory is that pressure is to volume as voltage is to charge, since for steady laminar flow along a straight pipe, Poiseuille's law is formally analogous to Ohm's law in defining a linear resistance. This analogy is extended to transients by considering the response to a step in the applied pressure difference. Although the exact solution [3, $\S 4.3]$ shows that the system has infinitely many degrees of freedom each with its own time-constant, the initial rate of change of flow-rate is proportional to the step in pressure, thereby defining a hydraulic analogue of inductance called inertance $[18,1]$.

Many circuit models are limited to 'two-terminal elements' [19], following Kirchhoff's laws and graph theory; however, lumped networks have been generalized to $n$-port components with the resistance and inertance of a twoended branch replaced by matrices of order $n$ [12]. The need for multiport components is characteristic of microhydraulics. Electrical circuits are more easily designed than microhydraulic systems to physically resemble graphs. The pressure drops in larger-scale hydraulic networks are conventionally classified into 'major losses' proportional to the length of long pipes and 'minor losses' induced by turns and junctions, with the major dominating the minor [20, §18]. However, microhydraulic systems intrinsically have many turns and junctions in a restricted space and so are less well modelled by graphs with pressures at ports and pressure drops along branches.

A simple example involving multiport inertance is a single ink-jet consisting of a nozzle through which the ink is ejected, an opening for refill, and an actuator [16]. In designing such a device, one wants to know how much of the work done by the actuator is wasted due to fluid travelling backwards up the supply line and how the backward inertance affects the forward flow. The circuit of Beasley [4] has three ports (supply, actuator, and nozzle) connected by two branches in the configuration supply-actuator-nozzle. The pressure is 
specified at the actuator as a function of time and the nozzle and supply are at fixed pressures; however, in such a circuit, the flow forward from actuator to nozzle depends only on the inertance of the branch between them and not on that back to the supply. Therefore the naïve two-terminal branched network model provides no answer to questions concerning backwards inertance.

\section{Theory of hydraulic inertance}

Whereas resistance is defined by the steady state, inertance characterizes the immediate response to a pressure impulse in an incompressible fluid. Here the classical theory of impulsively driven flow $[14, \S 11,3, \S 6.10]$ is recalled and the coefficients defined.

\subsection{Short-time impulse-response of incompressible fluid}

The velocity $\mathbf{u}$ and pressure $p$ of a fluid of constant density $\rho$ and viscosity $\mu$ are governed by the Navier-Stokes equations

$$
\begin{aligned}
\rho\left(\frac{\partial \mathbf{u}}{\partial t}+\mathbf{u} \cdot \nabla \mathbf{u}\right) & =-\nabla p+\mu \nabla^{2} \mathbf{u} \\
\nabla \cdot \mathfrak{u} & =0
\end{aligned}
$$

If forces on the boundary contain an impulsive part proportional to Dirac's $\delta(t)$, then for short times the pressure throughout must have the form $p(x, t) \sim$ $\Pi(\boldsymbol{x}) \delta(t)$, where $\Pi$ is the pressure impulse. From the integral of (1) over a time which tends to zero, it follows that if the velocity is to remain finite, it must have the form $\mathbf{u}(\boldsymbol{x}, \mathbf{t}) \sim \mathbf{U}(\boldsymbol{x}) \mathrm{H}(\mathbf{t})+\mathrm{O}(\mathbf{t})$, where $\mathrm{H}$ is Heaviside's step 
function and $\mathbf{U}$ and $\Pi$ satisfy [14, eq. 11.2, 3, eq. 6.10.2]

$$
\begin{aligned}
\rho \mathbf{U} & \sim-\nabla \Pi, \\
\nabla \cdot \mathbf{U} & =0 .
\end{aligned}
$$

\subsection{The multiport boundary value problem}

Consider the initial-boundary value problem for (1) in which the stagnant fluid $\mathbf{u}=0$ in $\Omega$ is contained within a rigid surface $\Gamma=\partial \Omega$ pierced by $\boldsymbol{n}$ 'ports' $\Gamma_{0}, \Gamma_{1}, \ldots, \Gamma_{n-1}$ at which a spatially uniform temporally varying pressure is specified and the flow is constrained to be normal to the surface. In particular, consider the response of the fluid to a set of impulsive pressures $\left.p\right|_{\Gamma_{k}}=\Pi_{k} \delta(t)$ for $k=0,1, \ldots, n-1$. The rest of the boundary, $\partial \Omega \backslash \cup_{j} \Gamma_{j}$, is constituted of impermeable walls.

Since the impulsive pressure $\left.\Pi\right|_{\Gamma_{k}}=\Pi_{k}$ is assumed uniform over each of the $\mathrm{n}$ ports, the space of flows is spanned by the $\mathrm{n}$ solutions obtained when each of the ports in turn gets a nonzero impulse while the rest are left at constant pressure; that is, for $\mathrm{k}=0,1, \ldots, \mathrm{n}-1$,

$$
\begin{aligned}
-\nabla^{2} \Pi^{(k)} & =0 & & \text { in } \Omega, \\
\Pi^{(k)} & =\delta_{j k} \Pi_{k} & & \text { on } \Gamma_{j} \text { with } j=0,1, \ldots, n-1, \\
\mathbf{n} \cdot \nabla \Pi^{(k)} & =0 & & \text { on } \partial \Omega \backslash \cup_{j} \Gamma_{j} .
\end{aligned}
$$

\subsection{Reciprocal inertance matrix}

The multiport reciprocal inertance coefficients are defined as the volumetric flow-rate through one port due to a unit impulse at another; that is,

$$
s_{i j} \equiv-\frac{1}{\Pi_{j}}\left(\mathbf{n}, \mathbf{u}^{(j)}\right)_{\Gamma_{i}} \equiv-\frac{1}{\Pi_{j}} \int_{\Gamma_{i}} \mathbf{n} \cdot \mathbf{u}^{(j)} \mathrm{d} \Gamma,
$$


where $\mathbf{n}$ is the outward unit normal, $\mathbf{u}^{(\mathfrak{j})}$ is derived from $\Pi^{(j)}$ by (3), and the $(\cdot, \cdot)$ denotes the inner product of two fields over the whole or part of the domain or boundary (here, as indicated by the subscript, just the ith port).

Given the application of $n$ different impulses at each of the ports, the combined impulsively generated velocity is $\mathbf{U}=\sum_{\mathbf{j}} \mathbf{U}^{(\mathbf{j})}$ and the corresponding volumetric flow-rate through the ith port is

$$
(\mathbf{n}, \mathbf{U})_{\Gamma_{i}}=\sum_{j=0}^{n-1} s_{i j} \Pi_{j} .
$$

Section 2.5 explains the need for reciprocal inertance.

\subsection{Two ways to calculate reciprocal inertance}

The reciprocal inertance coefficients are obtained from (8) and (3) as

$$
s_{i j} \equiv \frac{1}{\rho \Pi_{j}}\left(\mathbf{n}, \nabla \Pi^{(j)}\right)_{\Gamma_{i}}
$$

as shown by Asai [1, eq. 17] for the special case $n=2$ where the inertance is $s_{00}^{-1}$. Integrating by parts over the domain, the scalar product of two of the solutions for velocity is

$$
\begin{aligned}
\left(\mathbf{u}^{(i)}, \mathbf{u}^{(j)}\right)_{\Omega} & =\frac{1}{\rho}\left(\nabla \Pi^{(i)}, \mathbf{u}^{(j)}\right)_{\Omega} \\
& =\frac{1}{\rho}\left(\Pi^{(i)} \mathbf{n}, \mathbf{u}^{(j)}\right)_{\partial \Omega}-\frac{1}{\rho}\left(\Pi^{(i)}, \nabla \cdot \mathbf{u}^{(j)}\right)_{\Omega} .
\end{aligned}
$$

Simplifying using (4), (7), and (6) and introducing the definition (8) yields a second formula for the coefficients:

$$
s_{i j}=\frac{\rho\left(\mathbf{u}^{(i)}, \mathbf{u}^{(j)}\right)_{\Omega}}{\Pi_{i} \Pi_{j}} \equiv \frac{\rho \int_{\Omega} \mathbf{u}^{(i)} \cdot \mathbf{u}^{(j)} \mathrm{d} \Omega}{\Pi_{i} \Pi_{j}},
$$

which is equivalent to identifying $\frac{1}{2} \sum_{i j} \Pi_{i} s_{i j} \Pi_{j}$ with the total kinetic energy. 


\subsection{Properties of the reciprocal inertance matrix}

Symmetry of the reciprocal inertance matrix follows directly from (13). It follows from the maximum principle [14, §37] that the diagonal must be positive and the off-diagonals negative.

Besides the maximum principle, other classical theorems on potential flow imply other properties: that the columns [14, eq. 36.2] and rows [14, §40 $\gamma]$ of the matrix sum to zero, physically corresponding to incompressibility and the irrelevance of absolute pressure-impulse as opposed to differences in pressureimpulse, respectively. Thus the matrix is singular, which is why we work with reciprocal inertance rather than inertance.

\section{Discretization}

The Galerkin finite element method is convenient for its generality in handling complicated industrial geometry, but it is chosen in particular here since it leads to a novel third way to compute reciprocal inertance.

Assume that the impulsive pressures can be expanded in some basis

$$
\Pi^{(k)}(x)=\sum_{j=0}^{N-1} \phi_{j}(x) \Pi_{j}^{(k)},
$$

then the Galerkin equations are

$$
\sum_{j=0}^{N-1} a_{i j} \Pi_{j}^{(k)}=\sum_{j=0}^{N-1} \sum_{p=0}^{n-1}\left(\phi_{i} \mathbf{n}, \nabla \phi_{j}\right)_{\Gamma_{p}} \Pi_{j}^{(k)}
$$

where

$$
\mathrm{a}_{\mathrm{ij}} \equiv\left(\nabla \phi_{\mathrm{i}}, \nabla \phi_{\mathrm{j}}\right)_{\Omega}
$$


For a nodal basis with the subset $\mathcal{D}$ of indices corresponding to ports, the Galerkin equations (15) are partitioned; for degrees of freedom $i \in \mathcal{D}$, the equations are ignored since $\Pi_{i}^{(k)}$ is prescribed by (6); for $i \notin \mathcal{D}$, the right-hand sides vanish since $\phi_{i}=0$ on the ports. Thus

$$
\begin{aligned}
& \sum_{j=0}^{N-1} a_{i j} \Pi_{j}^{(k)}=0, \quad i \notin \mathcal{D} \\
& \sum_{j \notin D} a_{i j} \Pi_{j}^{(k)}=-\sum_{j \in D} a_{i j} \Pi_{j}^{(k)}, \quad i \notin \mathcal{D},
\end{aligned}
$$

which is a standard way of handling 'essential' (Dirichlet) conditions [6, §8.4.2].

\subsection{A third way to compute reciprocal inertance}

Given the expansion (14), equation (13) is discretized as

$$
s_{i j}=\frac{\sum_{r s} \Pi_{r}^{(i)} a_{r s} \Pi_{s}^{(j)}}{\rho \Pi_{i} \Pi_{j}} .
$$

This is particularly convenient if a nodal Galerkin method is used since then the coefficients (16) will be already available and (19) involves just a matrix-vector product and a scalar product. Unlike (10) or (13), no numerical differentiation of the potential is required to obtain to obtain the velocity.

As both $a_{i j}$ and $s_{i j}$ are coefficients which convert pressure-impulses into flowrates, (19) is interpreted as a Kron reduction [5], condensing the finite element stiffness matrix of the finite element nodes to the reciprocal inertance matrix of the ports of the vessel. This works by eliminating the nodes on the walls and interior of the finite element mesh and combining those on the ports into a single supernode for each port, giving a lumped model relating the pressure-impulses on the ports to the volumetric influx through them. 


\section{Implementation}

\subsection{Pseudocode}

The method was implemented with scikit-fem [10], a Python library written at a high enough level for the listings to also serve as pseudocode.

Given a mesh of a vessel in which the ports are marked, for example, from Gmsh [8], one selects a basis (14), for example the $\mathcal{P}_{1}$ basis of piecewise-linear Lagrange elements,

\section{basis = InteriorBasis (mesh, ElementTriP1())}

assembles the stiffness matrix (16),

$\mathrm{a}=\operatorname{asm}($ laplace, basis)

identifies the nodes lying on ports and therefore subject to essential conditions, ports = basis.get_dofs (mesh.boundaries)

identifies the complementary nodes corresponding to the degrees of freedom (17),

dofs = basis.complement_dofs(ports)

prefills the $\mathrm{n}$ solution vectors of length $\mathrm{N}$ with the Dirichlet data,

$p=\operatorname{zeros}(($ basis. $N$, len(mesh.boundaries $)))$

for $k$, port in enumerate(ports.values()):

$\mathrm{p}[$ port.all ()$, \mathrm{k}]=1$.

and solves for all $\mathfrak{n}$ right-hand sides of (18) simultaneously.

$\mathrm{p}[\mathrm{dofs}]=\operatorname{solve}(* \operatorname{condense}(\mathrm{a}, \operatorname{zeros}$ like $(\mathrm{p}), \mathrm{p}$, dofs $))$

The reciprocal inertance matrix is computed by the third method (19). 


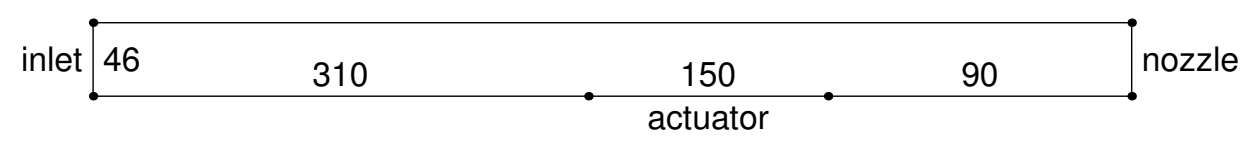

Figure 1: Two-dimensional version of the prototype bubble jet of Asai [2] with the three ports named and key dimensions marked in arbitrary units.

$\mathrm{s}=\mathrm{p} \cdot \mathrm{T} @ \mathrm{a} @ \mathrm{p}$

\subsection{Example: Asai's prototype bubble jet}

Asai [2] presented transient simulations of the actuation of a three-port bubble jet topologically equivalent to that discussed in Section 1 . Here a twodimensional version is used for demonstration; see Figure 1 for the dimensions and Figure 2 for the three pressure impulse fields. The reciprocal inertance matrix as extrapolated from the sequence of refined grids is (with ports ordered actuator, nozzle, inlet)

$$
\left[\begin{array}{lll}
s_{00} & s_{01} & s_{02} \\
s_{10} & s_{11} & s_{12} \\
s_{20} & s_{21} & s_{22}
\end{array}\right]=\left[\begin{array}{lll}
+0.361223 & -0.221071 & -0.140152 \\
-0.221071 & +0.221170 & -0.000099 \\
-0.140152 & -0.000099 & +0.140252
\end{array}\right] \text {. }
$$

The smallness of $s_{12}=s_{21} \approx-10^{-4}$ indicates that the two-branch model [4] is reasonable; nozzle-flows are little influenced by the inlet.

\subsection{Convergence}

The error of each of the three estimates for the $s_{01}$ actuator-nozzle coefficient is plotted in Figure 3 against $h$, the length of the longest edge in the mesh. An analysis is outside the present scope but a few features deserve comment. 
(a)

(b)

(c)

Figure 2: Pressure impulses for the (a) actuator, (b) nozzle, and (c) inlet.

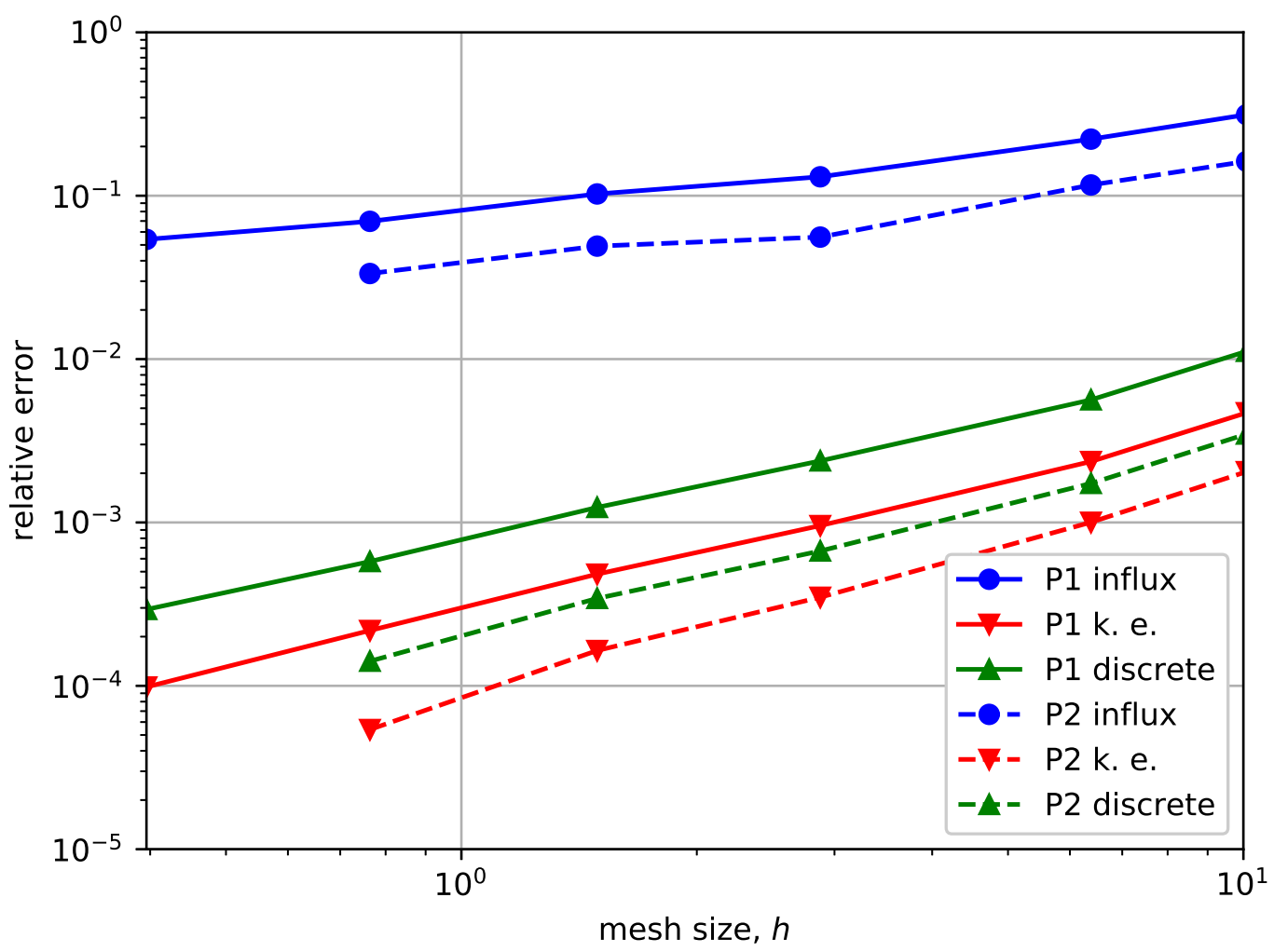

Figure 3: Relative error of $s_{01}$ with $\mathcal{P}_{1}$ or $\mathcal{P}_{2}$ elements, and based on: (blue) influx (10); (red) kinetic energy (13); or (green) the discrete quadratic form (19). 
The $\mathcal{P}_{2}$ piecewise-quadratic basis solutions converge no faster than the $\mathcal{P}_{1}$ piecewise-linear basis solutions. The actuator port is flush with the boundary whereas ideally ports are at right-angles to walls. Locally this induces a square-root singularity $[6, \S 3.1 .3]$ which lies in the Sobolev space $\mathrm{H}^{\sigma}$ only for $\sigma<3 / 2[9]$, limiting the finite element $H^{1}$ convergence of the $\Pi^{(k)}$, and so the $\mathrm{L}^{2}$ convergence of its gradient, to $\mathrm{o}(\sqrt{\mathrm{h}})$ [6, eq. 3.21]. This convergence is achieved by any piecewise degree $n$ polynomial basis $\mathcal{P}_{n}$ with $n>\sigma-1$, so $\mathcal{P}_{1}$ suffices.

The naïve estimate of inertance from its definition in terms of influx (10) is seen to converge most slowly; its observed rate $o(\sqrt{h})$ is just that of the error expected of the gradient of the potentials. Note that this is not $O(\sqrt{h})$ since the inequality $\sigma<3 / 2$ is strict.

The second (13) and third (19) methods are closely related and converge like the squared $H^{1}$-norm of the potentials, thus with $o(h)$, this $h$ being the square of the $\sqrt{h}$ limiting the potentials. This holds for any piecewise degree $n$ polynomial space $\mathcal{P}_{n}$ with $n \geqslant 1$.

From experience, although the second and third methods converge with the same order, the second is usually found to be more accurate in the preexponential factor perhaps as it involves 'gradient-averaging' which often leads to a more accurate approximation of the gradient [13].

\section{Conclusion}

Lumped models of microhydraulic vessels based on two-port branch circuits can be improved by the use of multiport components, replacing the scalar branch inertance with a reciprocal inertance matrix which is symmetric, has positive diagonal, negative off-diagonals, and zero row- and column-sums.

Classical $\mathcal{P}_{1}$ finite elements provide a simple method of computing the potential flow in multiport microhydraulic vessels. They are robust against 
the singularities not uncommon in received geometries. Of the three ways to postprocess the reciprocal inertance coefficients, the two based on energy generally converge faster than the basic boundary fluxes.

Acknowledgement We thank Frédéric Hecht for hints on (13) for $\mathfrak{n}=\mathbf{2}$ and an anonymous reviewer for insights on the errors (§ 4.3).

\section{References}

[1] A. Asai. "Bubble dynamics in boiling under high heat flux pulse heating". In: J. Heat Transf. 113.4 (1991), pp. 973-979. DOI: 10.1115/1.2911230 (cit. on pp. C142, C145).

[2] A. Asai. "Three-dimensional calculation of bubble growth and drop ejection in a bubble jet printer". In: J. Fluids Eng. 114.4 (1992), pp. 638-641. DOI: 10.1115/1.2910079 (cit. on p. C149).

[3] G. K. Batchelor. An introduction to fluid dynamics. Cambridge University Press, 1967. DOI: 10.1017/CB09780511800955 (cit. on pp. C142, C143, C144).

[4] J. D. Beasley. "Model for fluid ejection and refill in an impulse drive jet". In: Soc. Photogr. Sci. Eng. 21.2 (1977), pp. 78-82 (cit. on pp. C142, C149).

[5] F. Dörfler and F. Bullo. "Kron reduction of graphs with applications to electrical networks". In: IEEE T. Circuits I 60.1 (2013), pp. 150-163. DOI: 10.1109/tcsi.2012.2215780 (cit. on p. C147).

[6] A. Ern and J.-L. Guermond. Theory and practice of finite elements. Springer, 2004. DOI: 10.1007/978-1-4757-4355-5 (cit. on pp. C147, C151). 
[7] K. Foster and G. A. Parker. Fluidics: Components and circuits. Wiley, 1970. URL: https://www.worldcat.org/title/fluidicscomponents-and-circuits/oclc/138528 (cit. on p. C141).

[8] C. Geuzaine and J.-F. Remacle. "Gmsh: A 3-D finite element mesh generator with built-in pre- and post-processing facilities". In: Int. J. Numer. Meth. Eng. 79.11 (2009), pp. 1309-1331. DOI: 10.1002/nme. 2579 (cit. on p. C148).

[9] G. Geymonat and D. Chenais. "Introduction". In: Partial differential equations and functional analysis. Ed. by J. Cea, D. Chenais, G. Geymonat, and J. L. Lions. Birkhäuser, 1996, pp. xi-xii. DOI: 10.1007/978-1-4612-2436-5 (cit. on p. C151).

[10] T. Gustafsson and G. McBain. kinnala/scikit-fem 0.1.17. Nov. 2018. DOI: $10.5281 / z e n o d o .1490338$ (cit. on p. C148).

[11] O. Heaviside. "Some remarks on the Volta force and seat of electro-motive forces questions, and on impressed force and potential in condenser circuits". In: J. Soc. Tele.-Eng. Electric. 14.57 (1885), pp. 269-296. DOI: 10.1049/jste-3.1885.0014 (cit. on p. C142).

[12] H. E. Koenig, Y. Tokad, and H. K. Kesavan. Analysis of discrete physical systems. McGraw-Hill, 1967. URL: https://trove.nla.gov. $\mathrm{au} /$ work/21368152? selectedversion=NBD2690879 (cit. on p. C142).

[13] M. Kř́žzek and P. Neittaanmäki. "Superconvergence phenomenon in the finite element method arising from averaging gradients". In: Numer. Math. 45.1 (1984), pp. 105-116. DOI: $10.1007 /$ bf 01379664 (cit. on p. C151).

[14] H. Lamb. Hydrodynamics. 6th. Cambridge University Press, 1932, pp. 10-11. URL: https://www. cambridge.org/au/academic/ subjects/mathematics/fluid-dynamics-and-solidmechanics/hydrodynamics-6th-edition (cit. on pp. C143, C144, C146). 
[15] S. G. Mallinson et al. "Suppressing tiger stripes: Taming flow oscillations to improve print quality". In: 20th Australasian Fluid Mechanics Conference. 2016. URL: http://people.eng. unimelb.edu. au/imarusic/proceedings/20/478\%20Paper.pdf (cit. on p. C141).

[16] G. D. McBain and S. G. Mallinson. "Impulsively generated incompressible two-phase flow and the Asai thermal ink-jet model". In: 21st Australasian Fluid Mechanics Conference. 2018. URL: https://people.eng.unimelb.edu.au/imarusic/proceedings/21/ Contribution_616_final.pdf (cit. on p. C142).

[17] K. W. Oh, K. Lee, B. Ahn, and E. P. Furlani. "Design of pressure-driven microfluidic networks using electric circuit analogy". In: Lab Chip 12.3 (2012), pp. 515-545. DOI: 10.1039/c21c20799k (cit. on p. C141).

[18] H. F. Olson. Elements of acoustical engineering. Van Nostrand, 1948. DOI: 10.1002/sce. 3730320378 (cit. on pp. C141, C142).

[19] R. S. Sanford. Physical networks. Prentice-Hall, 1965. URL: https://trove.nla.gov .au/work/8815056?q\&versionId=10200776 (cit. on pp. C141, C142).

[20] V. L. Streeter. "Steady flow in pipes and conduits". In: Engineering hydraulics: Proceedings of the Fourth Hydraulics Conference. Ed. by H. Rouse. Wiley, 1950. Chap. 6, pp. 387-443. URL: https://trove.nla.gov.au/work/17727563 (cit. on p. C142).

\section{Author addresses}

1. G. D. McBain, Memjet, New South Wales 2113, Australia. mailto: gmcbain@memjet.com orcid:0000-0002-1904-122X

2. S. G. Mallinson, Memjet, New South Wales 2113, Australia. mailto:sam@memjet.com 
orcid:0000-0003-1323-1062

3. B. R. Brown, Memjet, New South Wales 2113, Australia.

4. Tom Gustafsson, Eniram, Helsinki, Finland mailto:tom.gustafsson@eniram.fi orcid:0000-0003-1611-5032 